\title{
Michaëlis-Menten kinetics: explicit dependence of substrate concentration on reaction time
}

\author{
by A. CRISTINA FREITAS and F. XAVIER MALCATA* \\ Escola Superior de Biotecnologia, Universidade Católica Portuguesa, \\ Rua Dr. António Bernardino de Almeida, 4200 Porto, Portugal
}

\begin{abstract}
The nonlinear dependence of the rate expressions associated with enzymecatalysed reactions on the concentration of substrate implies that the corresponding integrated form of the substrate mass balance in a batch reactor cannot be expressed as an explicit function of time..This paper addresses this problem for the classical case of Michaelis-Menten kinetics by providing a self-pacing exploration of the characteristics of a Taylor expansion of the substrate concentration on time. The accuracy of such an approximation is discussed. The procedure presented is appropriate to model situations of technological and practical interest.
\end{abstract}

\section{Introduction}

Enzymes, the functional units of cell metabolism, are remarkable catalysts because of their high specificity and activity towards their substrates at mild operating conditions. Since there is a decreasing trend in the cost of producing enzymes from microbial sources, enzymes are now available for industrial purposes in higher quantities and with higher purities. However, before being able to tackle the problem of designing enzyme reactors, one must postulate rate expressions and fit the associated rate parameters to experimental data often obtained in batch systems.

Traditionally, the kinetics of enzyme-catalysed reactions have been correlated by a rate expression derived from the Michaëlis-Menten mechanism [1]. This mechanism can be schematically represented as

$$
\mathrm{E}+\mathrm{S} \underset{k_{-1}}{\stackrel{k_{1}}{\rightleftarrows}} \mathrm{ES}^{\mathbf{k}_{\text {cat }}} \mathrm{E}+\mathrm{P}
$$

where $\mathrm{S}$ and $\mathrm{P}$ denote a substrate and a product molecule, respectively, $\mathrm{E}$ denotes an enzyme active site, ES denotes the enzyme/substrate complex, $k_{1}$ is a bimolecular intrinsic kinetic constant, and $k_{-1}$ and $k_{\text {cat }}$ are unimolecular intrinsic kinetic constants. The expression for the rate equation associated with this mechanism can be derived using either the quasi-steady-state or the rapid equilibrium approaches [2]. In either case the result reads

$$
r=\frac{V_{\max } C_{\mathrm{s}}}{K_{\mathrm{m}}+C_{\mathrm{s}}}
$$

where $r$ denotes the rate of production of product $\mathrm{P}$ and $C_{S}$ denotes the molar concentration of substrate. Here $v_{\max }$ is the rate under saturation conditions of

*'To whom all correspondence should be addressed. 
substrate (i.e. $v_{\max } \equiv k_{\mathrm{cat}} C_{\mathrm{E}}$, where $C_{\mathrm{E}}$ is the total concentration of enzyme active sites) and $K_{\mathrm{m}}$ is the Michaëlis-Menten constant (i.e. $K_{\mathrm{m}} \equiv k_{-1} / k_{1}$ using the rapid equilibrium approach, and $K_{\mathrm{m}} \equiv\left(k_{-1}+k_{\text {cat }}\right) / k_{1}$ using the quasi-steady-state approach). The material balance to substrate which is consumed by a chemical reaction in a well stirred batch reactor can be written as

$$
\begin{gathered}
-\frac{\mathrm{d} C_{S}}{\mathrm{~d} t}=r \\
C_{\mathrm{S}}\{t=0\}=C_{\mathrm{S}, 0}
\end{gathered}
$$

where $t$ denotes the time elapsed after start up of the reaction and $C_{s, 0}$ is the initial concentration of substrate. Combination of equation (2) and $(3 a)-(3 b)$ followed by integration via separation of variables yields

$$
t=\frac{C_{\mathrm{s}, 0}-C_{\mathrm{S}}}{v_{\max }}+\frac{K_{\mathrm{m}}}{v_{\max }} \ln \left\{\frac{C_{\mathrm{S}, 0}}{C_{\mathrm{s}}}\right\}
$$

which is of the form $t \equiv t\left\{C_{\mathrm{s}}\right\}$. Equation (4) can be rearranged to give

$$
t^{*}=1-C_{\mathrm{S}}^{*}-K_{\mathrm{m}}^{*} \ln \left\{C_{\mathrm{S}}^{*}\right\}
$$

where the dimensionless variables are defined as $t^{*} \equiv\left(v_{\max } t\right) / C_{\mathrm{S}, 0}, C_{\mathrm{S}}^{*} \equiv C_{\mathrm{s}} / C_{\mathrm{S}, 0}$, and $K_{\mathrm{m}}^{*} \equiv K_{\mathrm{m}} / C_{\mathrm{s}, 0}$.

For a number of purposes, this type of explicit dependence of the time ( $\left.t^{*}\right)$ on the concentration of substrate $\left(C_{\mathrm{S}}^{*}\right)$ is not convenient. One example pertains to the design of experiments involving enzyme-catalysed reactions: if one selects the $D$-optimal criterion [3], the variable associated with the larger error $\left(C_{S}^{*}\right)$ should be explicitly given as a function of the variable associated with the smaller error $\left(t^{*}\right)$ in order to obtain the Jacobians as univariate functions of the (desired) sampling times. Another example encompasses the nonlinear fitting of the rate expression to experimental data: the calculation of the predicted response, $C_{\mathrm{S}}^{*}$, at a given value of the predictor, $t^{*}$, requires either (i) solution of equation (5) by a trial and error technique, or (ii) numerical integration of equation (2) coupled with equations $(3 a)$ and $(3 b)$. Although convergent to the solution within a known number of steps, the former is a numerically inefficient procedure because each evaluation of the logarithmic function (computed in the CPU via a Taylor series) is time consuming. On the other hand, the latter requires involved quadrature methods able to provide a tight control or error propagation and CPU requirements which are difficult to anticipate. In both cases, it is impossible to obtain an analytical expression relating the $C_{\mathbf{S}}^{*}$ explicitly with $t^{*}$.

In the above (and other) situations, it would be more advantageous to have an analytical approximation for the explicit dependence of the concentration of substrate on time (i.e. it would be helpful to ohtain $C_{\mathrm{S}}^{*} \equiv C_{\mathrm{S}}^{*}\left\{t^{*}\right\}$ ) which is simultaneously simple, numerically efficient, and mathematically accurate. One way of proceeding to achieve the aforementioned goal is to expand $C_{\mathrm{S}}^{*}\left\{t^{*}\right\}$ about $t^{*}=0$ using a Taylor series, as explored in the following problem.

\section{Problem statement}

(1) Show that when $C_{\mathrm{S}}^{*}$ is expanded as a Taylor series about $t^{*}=0$ each coefficient $a_{n}$ of the expansion can be obtained as $a_{n} \equiv a_{n}\left\{K_{\mathrm{m}}^{*}\right\}$. 
(2) Write each derivative $d^{n} C_{\mathrm{S}}^{*} / \mathrm{d} t^{* n}$ as a function of $C_{S}^{*}, n, K_{\mathrm{m}}^{*}$, and $b_{n, i}(1 \leqslant i<n)$, where $b_{n, i}$ denotes a positive integer, and compute the values of $b_{n, i}$ for $n=1$ to $n=8$.

(3) Find a recurrence relation relating $b_{n+1, i}$ with $b_{n, i-1}$ and $b_{n, i}$.

(4) Prove that the Taylor expansion is convergent when $0 \leqslant K_{\mathrm{m}}^{*} \leqslant 1$.

(5) Obtain the asymptotic behaviours of the Taylor series when $K_{\mathrm{m}}^{*}$ is very small (pseudo-zero-order) and when $K_{\mathrm{m}}^{*}$ is very large (pseudo-first-order).

(6) Determine the variation of the quadratic error and the maximum local error of the Taylor approximation with the number of terms of this expansion for various values of $K_{\mathrm{m}}^{*}$.

(7) Compare the CPU time required for a similar final error using (i) an interval halving technique, (ii) an integration by finite differences, and (iii) a Taylor expansion.

\section{Problem solution}

(1) The form of the Taylor series associated with $C_{\mathrm{S}}^{*}=C_{\mathrm{S}}^{*}\left\{t^{*}\right\}$ can be written as

$$
C_{\mathrm{S}}^{*}=\sum_{n=0}^{\infty} a_{n} t^{* n}
$$

where each of the $a_{n}$ coefficients may be calculated using the following relationship:

$$
a_{n} \equiv \frac{1}{n !}\left(\frac{\mathrm{d}^{n} C_{\mathrm{S}}^{*}}{\mathrm{~d} t^{* n}}\right)_{t^{*}=0}
$$

The first derivative of $C_{\mathrm{S}}^{*}$ with respect to $t^{*}$ can be easily obtained using the definition of a derivative as a quotient of differentials, namely

$$
\left(\frac{\mathrm{d} C_{\mathrm{S}}^{*}}{\mathrm{~d} t^{*}}\right)_{t^{*}=0} \equiv \frac{1}{\left(\frac{\mathrm{d} t^{*}}{\mathrm{~d} C_{\mathrm{S}}^{*}}\right)_{t^{*}=0}}
$$

Recalling equation (5), one obtains $C_{\mathrm{S}}^{*}=1$ when $t^{*}=0$. Therefore, equation (8) becomes

$$
\left(\frac{\mathrm{d} C_{\mathrm{S}}^{*}}{\mathrm{~d} t^{*}}\right)_{t^{*}=0} \equiv \frac{1}{\left(\frac{\mathrm{d} t^{*}}{\mathrm{~d} C_{\mathrm{S}}^{*}}\right)_{C_{\mathrm{S}=1}^{*}}}=-\left(\frac{C_{\mathrm{S}}^{*}}{C_{\mathrm{S}}^{*}+K_{\mathrm{m}}^{*}}\right)_{C_{\mathrm{S}=1}^{*}}
$$

The higher-order derivatives can be obtained to advantage by recalling the rules of chain differentiation

$$
\begin{aligned}
\left(\frac{\mathrm{d}^{n} C_{\mathrm{S}}^{*}}{\mathrm{~d} t^{* n}}\right)_{t^{*}=0} & =\frac{1}{\left(\frac{\mathrm{d} t^{*}}{\mathrm{~d} C_{\mathrm{S}}^{*}}\right)_{C_{\mathrm{S}}^{*}=1}} \frac{\mathrm{d}}{\mathrm{d} C_{\mathrm{S}}^{*}}\left\{\frac{\mathrm{d}^{n-1} C_{\mathrm{S}}^{*}}{\mathrm{~d} t^{* n-1}}\right\}_{C_{\mathrm{S}}^{*}=1} \\
& =-\left(\frac{C_{\mathrm{S}}^{*}}{C_{\mathrm{S}}^{*}+K_{\mathrm{m}}^{*}}\right)_{C_{\mathrm{S}}=1} \frac{\mathrm{d}}{\mathrm{d} C_{\mathrm{S}}^{*}}\left\{\frac{\mathrm{d}^{n-1} C_{\mathrm{S}}^{*}}{\mathrm{~d} t^{* n-1}}\right\}_{C_{S}^{*}=1} \quad n \geqslant 2
\end{aligned}
$$

Combination of equations (7), and (9a)-(9b) finally allows one to write $a_{n} \equiv a_{n}\left\{K_{\mathrm{m}}^{*}\right\}$. 
(2) Recalling equation ( $9 a$ ) and employing equation (9b) for consecutively higher values of $n$ starting with $n=2$, the results thus obtained for the derivatives are of the general form

$$
\begin{gathered}
\frac{\mathrm{d} C_{\mathrm{S}}^{*}}{\mathrm{~d} t^{*}}=-\frac{C_{\mathrm{S}}^{*}}{K_{\mathrm{m}}^{*}+C_{\mathrm{S}}^{*}} \\
\frac{\mathrm{d}^{n} C_{\mathrm{S}}^{*}}{\mathrm{~d} t^{* n}}=K_{\mathrm{m}}^{*} \sum_{i=1}^{n-1} \frac{(-1)^{n+i-1} b_{n, i} C_{\mathrm{S}}^{* i}}{\left(K_{\mathrm{m}}^{*}+C_{\mathrm{S}}^{*}\right)^{n+i}} \quad n \geqslant 2
\end{gathered}
$$

where $b_{n, i}$ are positive integer coefficients. The numerical values of the $b_{n, i}$ coefficients for $n$ between 1 and 8 are listed in Table 1. The functional forms of the derivatives can then be easily obtained from combination of Table 1 and equations $(10 a)$ and $(10 b)$, which result in dependencies of the derivatives on $C_{\mathrm{S}}^{*}, n, K_{\mathrm{m}}^{*}$, and $b_{n, i}$.

(3) From the generic $n$ th-order derivative as given by equation $(10 b)$, one can obtain the next order derivative equation $(9 b)$, viz.

$$
\frac{\mathrm{d}^{n+1} C_{\mathrm{S}}^{*}}{\mathrm{~d} t^{* n+1}}=K_{\mathrm{m}}^{*} \sum_{i=1}^{n-1} \frac{(-1)^{n+i} b_{n, i} C_{\mathrm{S}}^{* i}}{\left(K_{\mathrm{m}}^{*}+C_{\mathrm{S}}^{*}\right)^{n+i+1}}\left(i-\frac{(n+i) C_{\mathrm{S}}^{*}}{K_{\mathrm{m}}^{*}+C_{\mathrm{S}}^{*}}\right) \quad n \geqslant 2
$$

Equation (11) may be rearranged to read

$$
\begin{gathered}
\frac{\mathrm{d}^{n+1} C_{\mathrm{S}}^{*}}{\mathrm{~d} t^{* n+1}}=K_{\mathrm{m}}^{*}\left(\frac{(-1)^{n+1} b_{n, 1} C_{\mathrm{S}}^{*}}{\left(K_{\mathrm{m}}^{*}+C_{\mathrm{S}}^{*}\right)^{n+2}}+\sum_{i=2}^{n-1}\left(\frac{(-1)^{n+i}(n+i-1) b_{n, i-1} C_{\mathrm{S}}^{* i}}{\left(K_{\mathrm{m}}^{*}+C_{\mathrm{S}}^{*}\right)^{n+i+1}}\right.\right. \\
\left.\left.+\frac{(-1)^{n+i} i b_{n, i} C_{\mathrm{S}}^{* i}}{\left(K_{\mathrm{m}}^{*}+C_{\mathrm{S}}^{*}\right)^{n+i+1}}\right)+\frac{(2 n-1) b_{n, n-1} C_{\mathrm{S}}^{* n}}{\left(K_{\mathrm{m}}^{*}+C_{\mathrm{S}}^{*}\right)^{2 n+1}}\right) \quad n \geqslant 2
\end{gathered}
$$

On the other hand, equation $(10 b)$ can be rewritten as

$$
\frac{\mathrm{d}^{n+1} C_{\mathrm{S}}^{*}}{\mathrm{~d} t^{* n+1}}=K_{\mathrm{m}}^{*} \sum_{i=1}^{n} \frac{(-1)^{n+i} b_{n+1, i} C_{\mathrm{S}}^{* i}}{\left(K_{\mathrm{m}}^{*}+C_{\mathrm{S}}^{*}\right)^{n+i+1}} \quad n \geqslant 1
$$

Comparing the forms of equations (12) and (13), one may obtain the coefficients

\begin{tabular}{|c|c|c|c|c|c|c|c|c|}
\hline$n$ & $i$ & 1 & 2 & 3 & 4 & 5 & 6 & 7 \\
\hline 2 & & 1 & & & & & & \\
\hline 3 & & 1 & 3 & & & & & \\
\hline 4 & & 1 & 10 & 15 & & & & \\
\hline 5 & & 1 & 25 & 105 & 105 & & & \\
\hline 6 & & 1 & 56 & 490 & 1260 & 945 & & \\
\hline 7 & & 1 & 119 & 1918 & 9450 & 17325 & 10395 & \\
\hline 8 & & 1 & 246 & 6825 & 56980 & 190575 & 270270 & 135135 \\
\hline
\end{tabular}
$b_{n+1, i}$ via the following recurrence relations:

$$
\begin{array}{cl}
b_{n+1,1}=b_{n, 1} & n \geqslant 1 \\
b_{n+1, i}=(n+i-1) b_{n, i-1}+i b_{n, i} & 2 \leqslant i \leqslant n-1 \quad n \geqslant 3 \\
\dot{v}_{n+1, n}=(2 n-1) b_{n, n-1} & n \geqslant 1
\end{array}
$$

Table 1. Num:rical values of the coefficients $b_{i, n}$. 
or, alternatively, for $n \geqslant 1, b_{n, 1}=1$ and

$$
b_{n, n-1}=\prod_{i=1}^{n-1}(2 i-1)
$$

All the aforementioned expressions are of the form $b_{n+1, i} \equiv b_{n+1, i}\left\{b_{n, i-1}, b_{n, i}\right\}$ for every value of $n$ and $i$.

(4) The ratio of any two consecutive terms of the Taylor series in question is given by

$\frac{a_{n+1} t^{* n+1}}{a_{n} t^{* n}} \equiv \frac{t^{*}\left(\frac{d^{n+1} C_{\mathrm{S}}^{*}}{d t^{* n+1}}\right)_{C_{\mathrm{S}=1}}}{(n+1)\left(\frac{d^{n} C_{\mathrm{S}}^{*}}{\mathrm{~d} t^{* n}}\right)_{C_{\mathrm{S}=1}}}=\frac{\sum_{i=1}^{n-1}(-1)^{n+i-1} \frac{b_{n, i}}{\left(1+K_{\mathrm{m}}^{*}\right)^{n+i}}\left(\frac{\left(n-i K_{\mathrm{m}}^{*}\right) t^{*}}{(n+1)\left(1+K_{\mathrm{m}}^{*}\right)^{2}}\right)}{\sum_{i=1}^{n-1}(-1)^{n+i-1} \frac{b_{n, i}}{\left(1+K_{\mathrm{m}}^{*}\right)^{n+i}}}$

where advantage was taken of equations $(10 b)$ and (11). The finite series in the numerator can be obtained from the corresponding series in the denominator by multiplying each term of the latter by $\left(n-i K_{\mathrm{m}}^{*}\right) t^{*} /\left[(n+1)\left(1+K_{\mathrm{m}}^{*}\right)^{2}\right]$. When $0 \leqslant K_{\mathrm{m}}^{*} \leqslant 1,\left(n-i K_{\mathrm{m}}^{*}\right)$ is always positive, so the following inequality can be easily derived:

$$
\frac{1-C_{\mathrm{S}}^{*}-\ln \left\{C_{\mathrm{S}}^{*}\right\}}{4(n+1)}<\frac{(n-i)\left(1-C_{\mathrm{S}}^{*}-\ln \left\{C_{\mathrm{S}}^{*}\right\}\right)}{4(n+1)} \leqslant \frac{\left(n-i K_{\mathrm{m}}^{*}\right) t^{*}}{(n+1)\left(1+K_{\mathrm{m}}^{*}\right)^{2}} \leqslant \frac{n\left(1-C_{\mathrm{S}}^{*}\right)}{n+1}
$$

where $t^{*}$ is eliminated via equation (5). If equation (16) holds, then the following relations can be written

$$
\begin{aligned}
& \frac{1-C_{\mathrm{S}}^{*}-\ln \left\{C_{\mathrm{S}}^{*}\right\}}{4(n+1)}=\frac{\left|\frac{1-C_{\mathrm{S}}^{*}-\ln \left\{C_{\mathrm{S}}^{*}\right\}}{4(n+1)}\right| \sum_{i=1}^{n-1} \frac{(-1)^{n+i-1} b_{n, i}}{\left(1+K_{\mathrm{m}}^{*}\right)^{n+i}} \mid}{\left|\sum_{i=1}^{n-1} \frac{(-1)^{n+i-1} b_{n, i}}{\left(1+K_{\mathrm{m}}^{*}\right)^{n+i}}\right|} \\
& \leqslant \frac{\left|\frac{\left(n-i K_{\mathrm{m}}^{*}\right) t^{*}}{(n+1)\left(1+K_{\mathrm{m}}^{*}\right)^{2}}\right|\left|\sum_{i=1}^{n-1} \frac{(-1)^{n+i-1} b_{n, i}}{\left(1+K_{\mathrm{m}}^{*}\right)^{n+i}}\right|}{\left|\sum_{i=1}^{n-1} \frac{(-1)^{n+i-1} b_{n, i} \mid}{\left(1+K_{\mathrm{m}}^{*}\right)^{n+i}}\right|}=\frac{\left|a_{n+1} t^{* n+1}\right|}{\left|a_{n} t^{* n}\right|} \\
& \frac{\left|a_{n+1} t^{* n+1}\right|}{\left|a_{n} t^{* n}\right|}=\frac{\left|\frac{\left(n-i K_{\mathrm{m}}^{*}\right) t^{*}}{(n+1)\left(1+K_{\mathrm{m}}^{*}\right)^{2}}\right|\left|\sum_{i=1}^{n-1}(-1)^{n+i-1} \frac{b_{n, i}}{\left(1+K_{\mathrm{m}}^{*}\right)^{n+i}}\right|}{\left|\sum_{i=1}^{n-1}(-1)^{n+i-1} \frac{b_{n, i}}{\left(1+K_{\mathrm{m}}^{*}\right)^{n+i}}\right|} \\
& \leqslant \frac{\left|\frac{n\left(1-C_{\mathrm{S}}^{*}\right)}{n+1}\right|\left|\sum_{i=1}^{n-1}(-1)^{n+i-1} \frac{b_{n, i}}{\left(1+K_{\mathrm{m}}^{*}\right)^{n+i}}\right|}{\left|\sum_{i=1}^{n-1}(-1)^{n+i-1} \frac{b_{n, i}}{\left(1+K_{\mathrm{m}}^{*}\right)^{n+i}}\right|}=\frac{n\left(1-C_{\mathrm{S}}^{*}\right)}{n+1}
\end{aligned}
$$


(The use of absolute values is necessary in order to keep the signs of the inequalities because although for sufficiently small positive values of $K_{\mathrm{m}}^{*}$ the coefficients $a_{n}$ of the terms of the Taylor expansion are all positive, for larger values of $K_{\mathrm{m}}^{*}$ the values of $a_{n}$ tend to take positive and negative values almost at random.) Equation (17) is also valid for $n$ sufficiently large, so

$$
\begin{aligned}
0= & \lim _{n \rightarrow \infty}\left(\frac{1-C_{S}^{*}-\ln \left\{C_{S}^{*}\right\}}{4(n+1)}\right) \leqslant \lim _{n \rightarrow \infty} \frac{\left|a_{n+1} t^{* n+1}\right|}{\left|a_{n} t^{* n}\right|} \\
& \leqslant \lim _{n \rightarrow \infty}\left(\frac{n}{n+1}\left(1-C_{S}^{*}\right)\right)=1-C_{S}^{*}<1
\end{aligned}
$$

which is valid for all values of $C_{\mathrm{S}}^{*}$ with physical meaning (i.e. $0<C_{\mathrm{S}}^{*}<1$ ). According to d'Alembert's ratio test [4], this result implies that the series with generic term $\left|a_{n} t^{* n}\right|$ is convergent. Therefore, the series-with generic term $a_{n} t^{* n}$ is also convergent [4].

(5) Recalling equations (6), (7), and (9a), rewriting all terms in the summation in equation $(10 b)$ with a common denominator, and performing some algebraic work, one concludes that all the terms of the Taylor expansion are of the form

$$
\begin{aligned}
& a_{0}=1 \\
& a_{1}=-\frac{1}{1+K_{\mathrm{m}}^{*}} \\
& a_{n}=\frac{1}{n !} \frac{K_{\mathrm{m}}^{*}}{\left(1+K_{\mathrm{m}}^{*}\right)^{2 n-1}} \sum_{i=1}^{n-1}(-1)^{i-1} c_{n, i} K_{\mathrm{m}}^{* i-1} \quad n \geqslant 2
\end{aligned}
$$

where the positive integer coefficients, $c_{n, i}$, are tabulated in Table 2. From this table, it is obvious that $c_{n, 1}=(n-1)$ ! and $c_{n, n-1}=1$.

If $K_{\mathrm{m}}^{*} \ll C_{\mathrm{S}}^{*} \leqslant 1$, then $\left(1+K_{\mathrm{m}}^{*}\right) \sim 1$ and

$$
\sum_{i=1}^{n-1}(-1)^{i-1} c_{n, i} K_{\mathrm{m}}^{* i-1} \sim c_{n, 1}=(n-1) !
$$

so using equations (6) and (19a)-(19c) one obtains

$$
C_{\mathrm{S}}^{*} \sim 1-t^{*}+K_{\mathrm{m}}^{*} \sum_{n=2}^{\infty} \frac{t^{* n}}{n}
$$

Equation (20) is approximately equivalent to

$$
C_{\mathrm{s}}^{*}=1-t^{*}
$$

\begin{tabular}{rrrrrrrr}
\hline$n$ & \multicolumn{1}{c}{$\boldsymbol{i}$} & 1 & 2 & 3 & 4 & 5 & 6 \\
\hline 2 & 1 & & & & & & \\
3 & 2 & 1 & & & & & \\
4 & 6 & 8 & 1 & & & & \\
5 & 24 & 58 & 22 & 1 & & & \\
6 & 120 & 444 & 328 & 52 & 1 & 1 & \\
7 & 720 & 3708 & 4400 & 1452 & 114 & 240 & 1 \\
8 & 5040 & 33988 & 58140 & 32130 & 5610 & \\
\hline
\end{tabular}

Table 2. Numerical values of the coefficients $c_{i, n}$. 


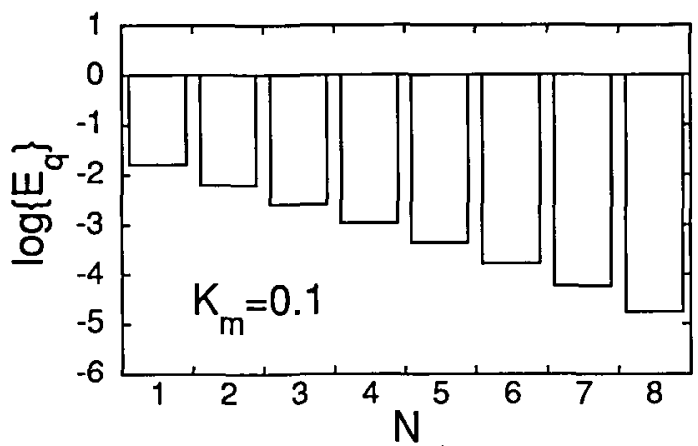

(a)

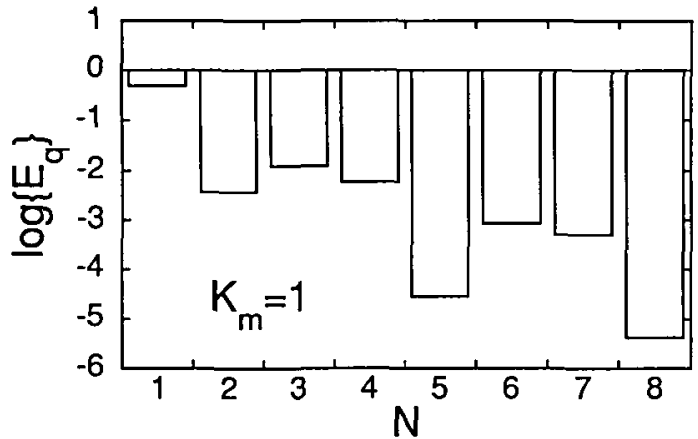

(b)

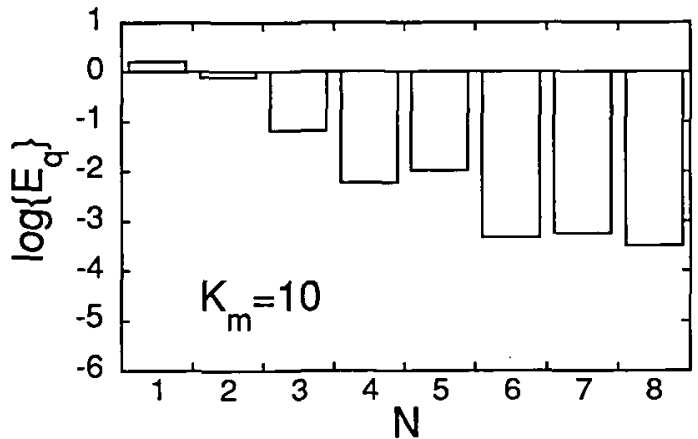

(c)

Figure 1. Log-lin plot of the integral quadratic error of the Taylor expansion, $E_{\mathrm{q}}$, as a function of $N$ for $(a) K_{\mathrm{m}}^{*}=0 \cdot 1,(b) K_{\mathrm{m}}^{*}=1$, and $(c) K_{\mathrm{m}}^{*}=10$, for $C_{\mathrm{S}, \mathrm{f}}^{*}=0 \cdot 1$.

because $K_{\mathrm{m}}^{*}$ is assumed to be vanishingly small. Equation (21) is formally equivalent to equation (5) when $K_{\mathrm{m}}^{*} \rightarrow 0$, i.e.

$$
t^{*}=1-C_{\mathrm{S}}^{*}
$$

If on the other hand, $K_{\mathrm{m}}^{*} \gg 1 \geqslant C_{\mathrm{S}}^{*}$, then $\left(1+K_{\mathrm{m}}^{*}\right) \sim K_{\mathrm{m}}^{*}$ and

$$
\sum_{i=1}^{n-1}(-1)^{i-1} c_{n, i} K_{\mathrm{m}}^{* i-1} \sim c_{n, 1} K_{\mathrm{m}}^{* n-2}=K_{\mathrm{m}}^{* n-2}
$$

so using equations (6) and $(19 a)-(19 c)$ one obtains

$$
C_{\mathrm{S}}^{*}=\sum_{n=0}^{\infty} \frac{(-1)^{n}}{n ! K_{\mathrm{m}}^{* n}} t^{* n}
$$




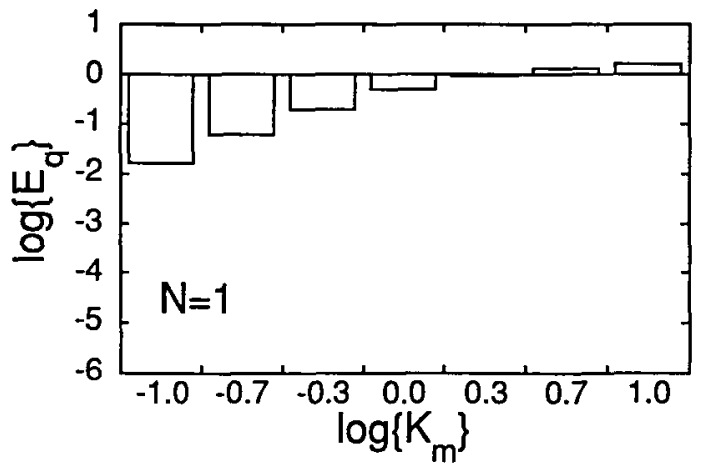

(a)

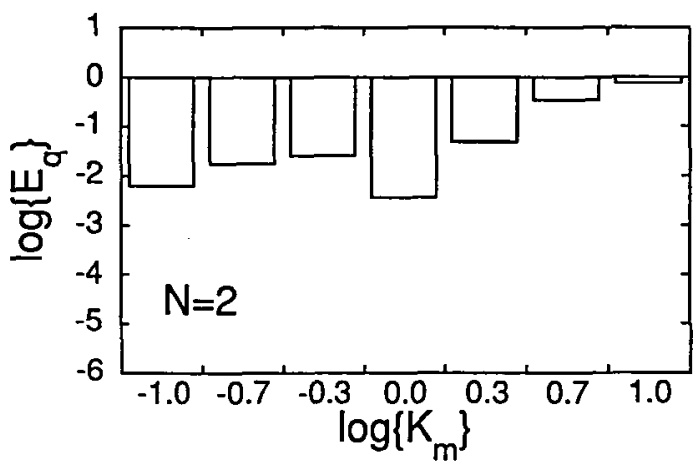

(b)

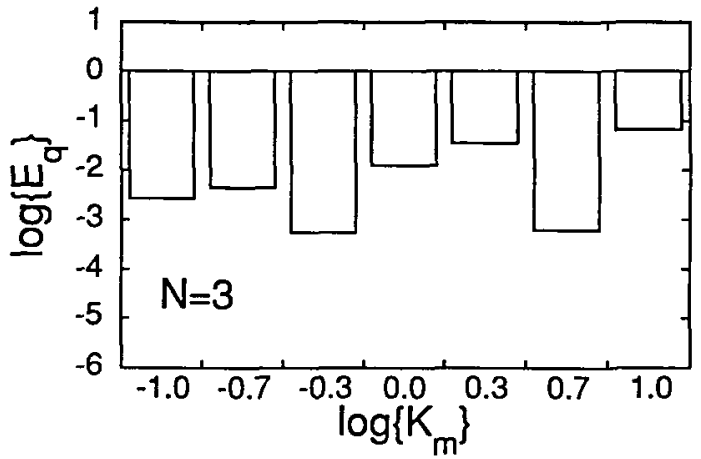

(c)

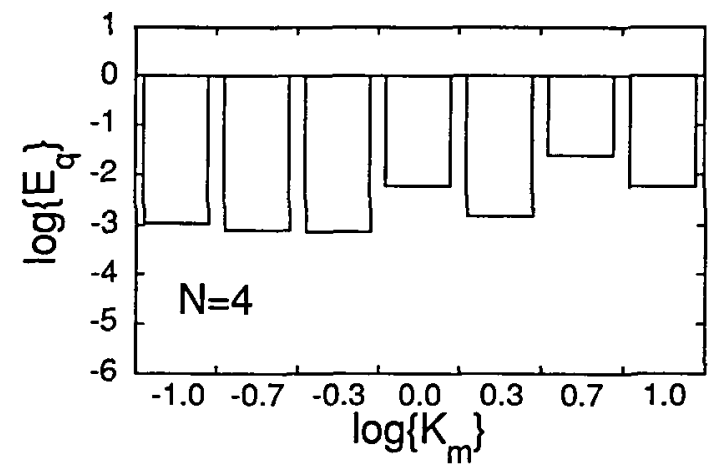

(d) 


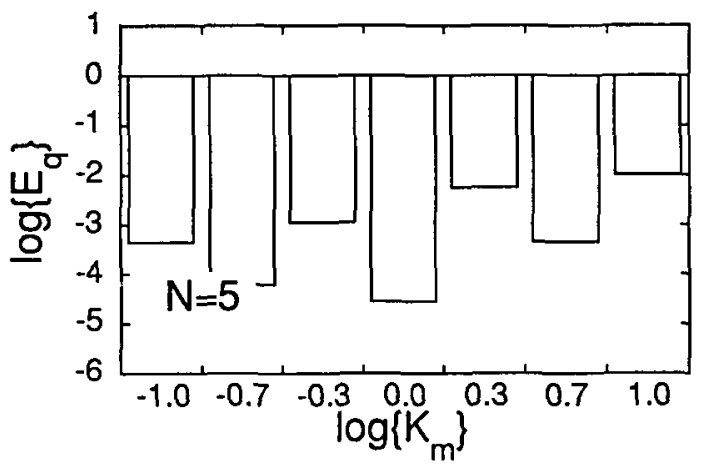

(e)
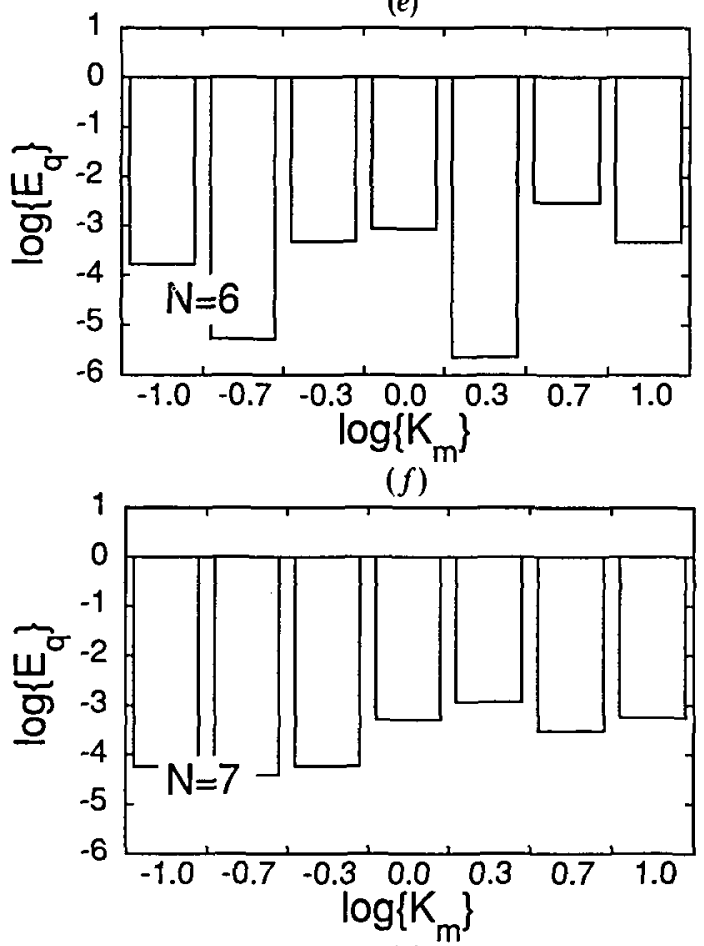

$(g)$

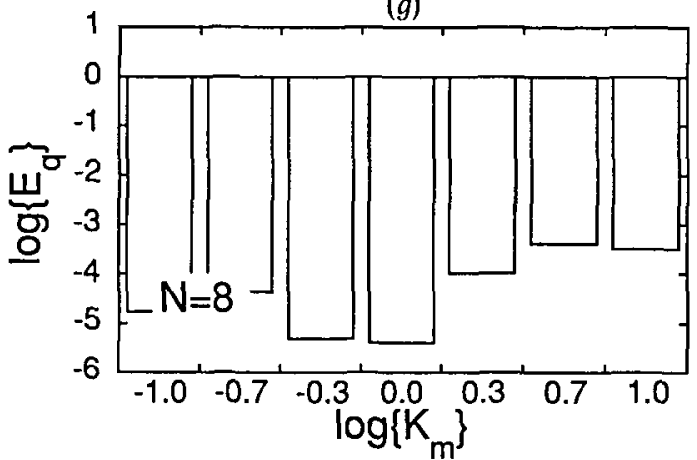

(h)

Figure 2. $\log -\log$ plot of the integral quadratic error of the Taylor expansion, $E_{\mathrm{q}}$, as a function of $K_{\mathrm{m}}^{*}$ for $(a) N=1,(b) N=2,(c) N=3,(d) N=4,(e) N=5,(f) N=6,(g) N=7$, and $(h) N=8$ for $C_{\mathrm{S}, \mathrm{f}}^{*}=0 \cdot 1$. 
where the right-hand side is the Taylor expansion of an exponential function, namely

$$
C_{\mathrm{S}}^{*}=\exp \left\{-\frac{t^{*}}{K_{\mathrm{m}}^{*}}\right\}
$$

Rearranging equation (24), one gets

$$
t^{*}=-K_{\mathrm{m}}^{*} \ln \left\{C_{\mathrm{S}}^{*}\right\}
$$

which is formally equivalent to equation (5) when $K_{\mathrm{m}}^{*} \rightarrow \infty$.

(6) The integral quadratic error of the Taylor expansion, $E_{\mathrm{q}}$, truncated after the $N$ th term, defined as

$$
\begin{array}{r}
E_{\mathrm{q}} \equiv \int_{C_{\mathrm{S}, \mathrm{f}}^{*}}^{1}\left(\left(\sum_{n=0}^{N} a_{n} t^{* n}\right)-C_{\mathrm{S}}^{*}\right)^{2} \mathrm{~d} C_{\mathrm{S}}^{*}= \\
\int_{C_{\mathrm{S}, \mathrm{f}}}^{1}\left(\left(\sum_{n=0}^{N} a_{n}\left(1-C_{\mathrm{S}}^{*}-K_{\mathrm{m}}^{*} \ln \left\{C_{\mathrm{S}}^{*}\right\}\right)^{n}\right)-C_{\mathrm{S}}^{*}\right)^{2} \mathrm{~d} C_{\mathrm{S}}^{*}=
\end{array}
$$

is plotted in figure 1 as a function of $N$ for various values of $K_{\mathrm{m}}^{*}$ and in figure 2 as a function of $K_{\mathrm{m}}^{*}$ for various values of $N$, in both cases with $C_{\mathrm{S}, \mathrm{f}}^{*}$ set arbitrarily equal to $0 \cdot 1$.

In a similar fashion, the maximum local error of the approximation, $E_{\mathrm{m}}$, obtained for $C_{\mathrm{s}}^{*}=C_{\mathrm{s}, \mathrm{f}}^{*}$ (or, equivalently, for $t^{*}=t_{\mathrm{f}}^{*}$ ) via truncation after the $N$ th term, defined as

$$
E_{\mathrm{m}} \equiv\left(\sum_{n=0}^{N} a_{n} t_{\mathrm{f}}^{* n}\right)-C_{\mathrm{S}, \mathrm{f}}^{*}=\left(\sum_{n=0}^{N} a_{n}\left(1-C_{\mathrm{S}, \mathrm{f}}^{*}-K_{\mathrm{m}}^{*} \ln \left\{C_{\mathrm{S}, \mathrm{f}}^{*}\right\}\right)^{n}\right)-C_{\mathrm{S}, \mathrm{f}}^{*}
$$

is plotted in figure 3 as a function of $N$ for various values of $K_{\mathrm{m}}^{*}$ when $C_{\mathrm{S}, \mathrm{f}}^{*}$ is set arbitrarily equal to $0 \cdot 1$.

As expected with a typical Taylor expansion, both the quadratic and the local maximum error of the approximation exhibit a decreasing trend as the number of terms in the series increases. When $K_{\mathrm{m}}^{*}$ tends to zero, both errors decrease exponentially with $N$; for larger values of $K_{\mathrm{m}}^{*}$, there are some fluctuations around this behaviour which result from the fact that the values of $a_{n}$ may take positive and negative values almost at random (see Table 2 and equation (19c)). In general the errors tend to decrease with increasing $K_{\mathrm{m}}^{*}$ because the exponential behaviour, which requires an infinite number of terms, is approached at the expense of a finite $N$. For $N$ equal to, say, 8 , both errors are sufficiently small to yield acceptable approximations for most practical purposes.

(7) Setting $t_{\mathrm{f}}^{*}$ and $K_{\mathrm{m}}^{*}$ arbitrarily equal to 23.926 and 10 , respectively, the exact value of $C_{\mathrm{S}, \mathrm{f}}^{*}$ is obtained from equation (5) to be $0 \cdot 1$ (these values correspond to the maximum errors $E_{\mathrm{q}}$ and $E_{\mathrm{m}}$ within an acceptable working range). The corresponding values of $C_{\mathrm{S}, \mathrm{f}}^{*}$ obtained from the Taylor approximation with the first 8 terms, $C_{\mathrm{S}, \mathrm{r}}^{*}\left\{t^{*}=t_{f}^{*}\right\}$, the interval halving technique with six iterations starting with the search interval $[0.008,0.992], C_{S}^{*}, \mathrm{~b}\left\{t^{*}=t_{\mathrm{f}}^{*}\right\}$, and the one-sided finite difference method with 15 steps from the initial condition $C_{\mathrm{S}}^{*}=1, C_{\mathrm{S}, \Delta}^{*}\left\{t^{*}=t_{\mathrm{f}}^{*}\right\}$ were calculated using a personal microcomputer. All these approaches yielded the same absolute error, \pm 0.082 . The average $\mathrm{CPU}$ times required to compute $C_{\mathrm{S}, \mathrm{T}}^{*}, C_{\mathrm{S}, \mathrm{b}}^{*}$, and $C_{\mathrm{S}, \Delta}^{*}$ were $11.54,14.97$, and $37.57 \mathrm{~ms}$, respectively. Therefore, the Taylor expansion is the most convenient method since it combines rapidity with accuracy. 


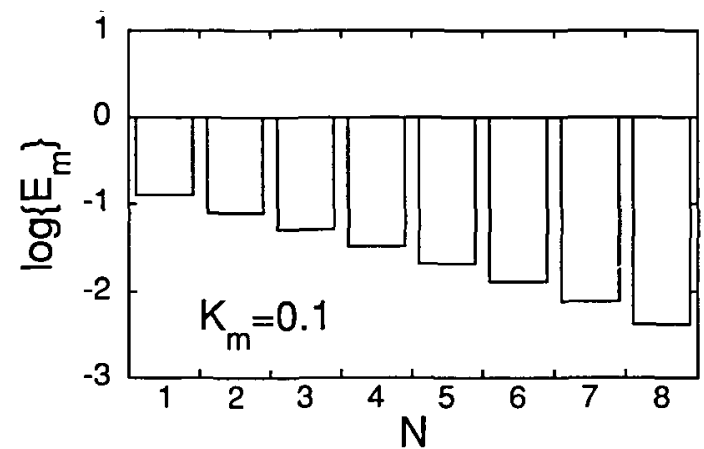

(a)

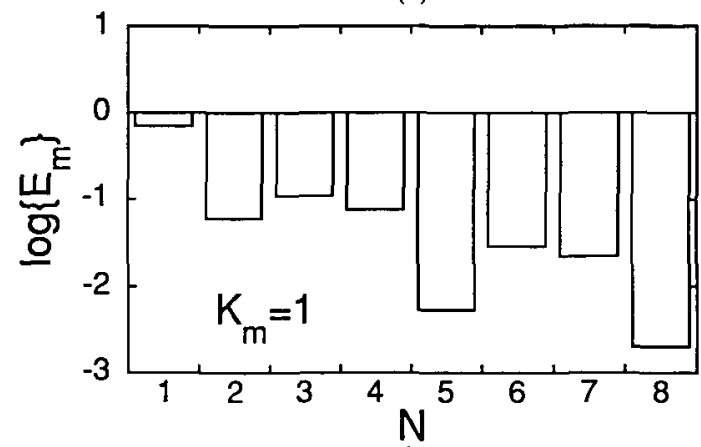

(b)

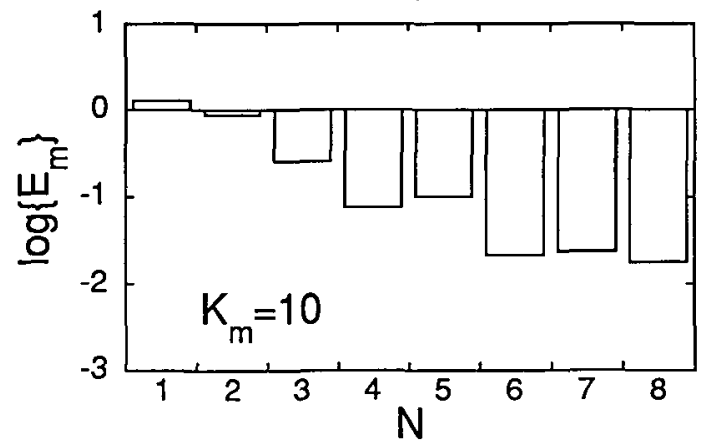

(c)

Figure 3. Log-lin plot of the local truncation error of the Taylor expansion, $E_{\mathrm{m}}$, as a function of $N$ for $(a) K_{\mathrm{m}}^{*}=0 \cdot 1,(b) K_{\mathrm{m}}^{*}=1$, and $(c) K_{\mathrm{m}}^{*}=10$ assuming that $C_{\mathrm{S}, \mathrm{f}}^{*}=0 \cdot 1$.

\section{Discussion}

Several modelling (and optimization) problems associated with the implicit dependence of the substrate concentration on the reaction time when the system is well described by the Michaellis-Menten rate expression have been discussed in the literature. Since there are no ciosed form analytical expressions for a number of quantities related to the Michaëlis-Menten rate expression (e.g. the efficiency factor), some authors have attempted to correlate the behaviour of such systems as combinations of the zero and first-order limiting behaviours of the aforementioned rate expression (for which there are simple, closed forms for such quantities). Examples include (i) expressing the efficiency factor of catalyst pellets containing a 
uniformly distributed immobilized enzyme as a combination of the efficiency factors calculated analytically for the asymptotic zero-order and first-order kinetics [5] and (ii) assessing the influence of diffusion limitations on the apparent rate of deactivation of enzymes in immobilized states [6]. In the present case, attempts to estimate the parameters of a linear combination of the zero and first-order explicit expressions for $C_{S}^{*} \equiv C_{S}^{*}\left\{t^{*}\right\}$ which yield the minimum value for either the integral error or the integral quadratic error over the tentative range $0 \cdot 1<C_{\mathrm{S}}^{*}<1$ failed to provide accurate approximants; hence the (more complex) Taylor approach was tested.

The Taylor expansion is convergent for all possible values of $C_{\mathrm{S}}^{*}$ between 0 and 1 , irrespective of the value of $K_{\mathrm{m}}^{*}$. Although this observation is easily consubstantiated via the computation of the consecutive terms of the expansion, the general mathematical proof of convergence is difficult when $K_{\mathrm{m}}^{*}>1$ because in this case $\left(n-i K_{m}^{*}\right)$ takes positive values when $i$ is small and negative values when $i$ is large (assuming that $n$ grows without limit). Hence, this subproblem was not considered here for the sake of simplicity.

It should be emphasized that the asymptotic case of pseudo-zero-order rate expression (i.e. small $K_{\mathrm{m}}^{*}$ ) requires only the linear term of the Taylor expansion, whereas the asymptotic case of pseudo-first-order rate expression (i.e. large $K_{\mathrm{m}}^{*}$ ) corresponds to an infinite number of terms, in a way that is formally equivalent to an explicit exponential dependence on $t^{*}$. For increasing intermediate values of $K_{\mathrm{m}}^{*}$, an increasing number of terms is necessary for a good approximation. The increasing number of terms required for a good approximation is, thus, conceptually derived from the fact that the Michaëlis-Menten rate expression is a variable order rate expression (which departs from zero order for high concentrations and evolves towards first order for small concentrations of substrate), where each additional term corresponds to an incremental increase in the apparent order of the reaction.

In general, the Taylor expansion provides the advantages of (i) being an analytical, explicit relationship of $C_{\mathrm{S}}^{*}$ on $t^{*}$, (ii) requiring a very small number of terms not only for convergence but also for good accuracy, and (iii) using such simple computational operations as additions and multiplications (which leads to small running times when compared with alternative numerical approaches). Therefore, it can be used safely in most practical applications where an enzyme-catalysed reaction is carried out in either a stirred batch reactor or a plug flow continuous reactor.

\section{Nomenclature}

$a_{n}=$ coefficient of the Taylor expansion

$b_{n}=$ auxiliary integer coefficient

$c_{n}=$ auxiliary integer coefficient

$C_{\mathrm{E}}=$ molar concentration of enzyme

$C_{\mathrm{S}}=$ molar concentration of substrate

$C_{\mathrm{S}, \mathrm{b}}=$ molar concentration of substrate computed using the internal halving method

$C_{\mathrm{S}, \mathrm{T}}=$ molar concentration of substrate computed using the Taylor expansion

$C_{\mathrm{S}, \Delta}=$ molar concentration of substrate computed using the finite difference technique

$\mathrm{E}=$ enzyme molecule

$\mathrm{ES}=$ enzyme/substrate complex

$E_{\mathrm{m}}=$ maximum local error of the Taylor expansion 
$E_{\mathrm{q}}=$ integral quadratic error of the Taylor expansion

$k_{1}=$ kinetic constant associated with binding of substrate to the enzyme

$k_{-1}=$ kinetic constant associated with dissociation of the enzyme/substrate complex

$k_{\text {cat }}=$ kinetic constant associated with transformation of the enzyme/substrate complex

$K_{\mathrm{m}}=$ Michaëlis-Menten constant

$\mathrm{P}=$ product molecule

$r=$ rate of reaction

$\mathrm{S}=$ substrate molecule

$t=$ time of reaction

$v_{\max }=$ reaction rate under saturating conditions of the enzyme with substrate

\section{Subscripts}

$0=$ at the initial conditions

$\mathrm{f}=$ at the final conditions

\section{Superscript}

*= dimensionless variable or parameter

\section{References}

[1] Bailey, J. E., and Ol.tis, D. F., 1975, Biochemical Engineering Fundamentals (New York: Wiley) pp. 100-103.

[2] Segel, I. H., 1975, Enzyme Kinetics (New York: Wiley), pp. 18-22.

[3] Bates, D. M., and Watrs, D. G., 1988, Nonlinear Regression Analysis and Its Applications (New York: Wiley) pp.122-129.

[4] Stephenson, G., 1973, Mathematical Methods for Science Students (London: Longman) pp. 71-82.

[5] Kobayashi, 'T., and Laidler, K. J., 1973, Biochem. Biophys. Acta, 302, 1.

[6] Yamane, T., Sirirote, P., and Shimizu, S., 1987, Biotechnol. Bioeng., 30, 963. 